\title{
HDL COLESTEROL. PROTECTOR DE ENFERMEDAD CARDIOVASCULAR: CALIDAD VS. CANTIDAD
}

\author{
HDL CHOLESTEROL. \\ CARDIOVASCULAR DISEASE PROTECTOR \\ QUALITY AGAINST QUANTITY
}

AGUSTÍN IZA STOLL

Universidad Norbert Wiener

\section{RESUMEN}

Los estudios pioneros de Framingham mostraron una relación inversa entre los niveles bajos de HDL Colesterol y la morbilidad coronaria. Los ensayos con modificaciones en el estilo de vida han mostrado un muy discreto incremento en las cifras de HDL-C, y la intervención farmacológica con fibratos o acido nicotínico (niacina) no ha mostrado tampoco disminución de la mortalidad. Tratar a pacientes con medicamentos que afectan el transporte reverso de colesterol y que aumentan considerablemente la cifra de HDL tampoco ha logrado disminuir la morbilidad. La asociación inversa de cifras altas de HDL y mortalidad coronaria está siendo cuestionada en los últimos años y se ha comenzado a investigar la calidad del HDL o la de sus subfracciones como explicación a su actividad antiaterosclerótica.

Palabras clave: HDL colesterol, CETP (cholesteryl ester transfer protein) morbimortalidad coronaria, transporte reverso de colesterol, número de partículas HDL, tamaño de partículas HDL.

\section{ABSTRACT}

The Framingham pioneers surveys shows an inverse relationship between the low levels of High Density Lipoprotein (HDL) cholesterol and coronary morbidity. The trials with modifications in the lifestyle have showed a very discrete increase in the figures of HDL-C and the pharmacological intervention with fibrates or nicotinic acid (niacin). They have also not showed a decrease of the mortality. Treat patients with medicines that affect the reverse transport of the cholesterol and that increase significantly the figures of HDL it neither has achieved to decrease the morbidity. The inverse association of high figures of HDL and the coronary mortality have been questioned in the last year and have already started to research the quality of HDL or their sub fraction like explanation to their antiatherosclerotic activities.

Key words: HDL cholesterol, CETP (Cholesteryl ester transfer protein), coronary morbidity and mortality, reverse transport of cholesterol, number of particles HDL, HDL particles Size. 


\section{INTRODUCCIÓN}

El colesterol normalmente tiene funciones muy importantes, especialmente en la constitución de las membranas celulares. Se transporta en la sangre en diversas formas, asociado a lipoproteínas de baja densidad (LDL por las siglas en inglés de low density lipoproteins) o lipoproteínas de alta densidad (HDL por las siglas en inglés de high density lipoprotein). Pero cuando hay mucho colesterol en la sangre, los estudios epidemiológicos extensos han mostrado una relación directa entre los niveles séricos de LDL colesterol y la morbi-mortalidad cardiovascular; asimismo, se ha demostrado que disminuyendo farmacológicamente los niveles de LDL, principalmente con estatinas, se consigue disminuir la morbilidad y la mortalidad cardiovascular ${ }^{1}$.

El LDL en niveles excesivos se deposita en la pared arterial, y en unión de otros componentes contribuye a formar la placa aterosclerótica, responsable de la enfermedad arterial; en cambio, el HDL, con un mecanismo de transporte reverso de colesterol, retira el colesterol de la placa y lo transporta al hígado, desde donde pasa a la bilis y al intestino para su excreción.

Desde los estudios pioneros del Framingham Heart Study (FHS), realizados hace varias décadas, se ha enfatizado que los niveles bajos en sangre de HDL no son protectores de la enfermedad vascular y se ha popularizado el sobrenombre de "colesterol bueno", a diferencia del LDL, al que se le llama "colesterol malo". Un primer reporte que demostró la relación inversa entre los niveles séricos de HDL-C y la incidencia de cardiopatía coronaria se basó en el seguimiento por 4 años de personas entre 49 y 82 años de edad, y uno posterior, con seguimiento de 12 años que incluyó a la mayoría de estos mismos pacientes, mostró

FIGURA 1

Asociación entre niveles de HDL-C y riesgo coronario de acuerdo a los niveles de colesterol total

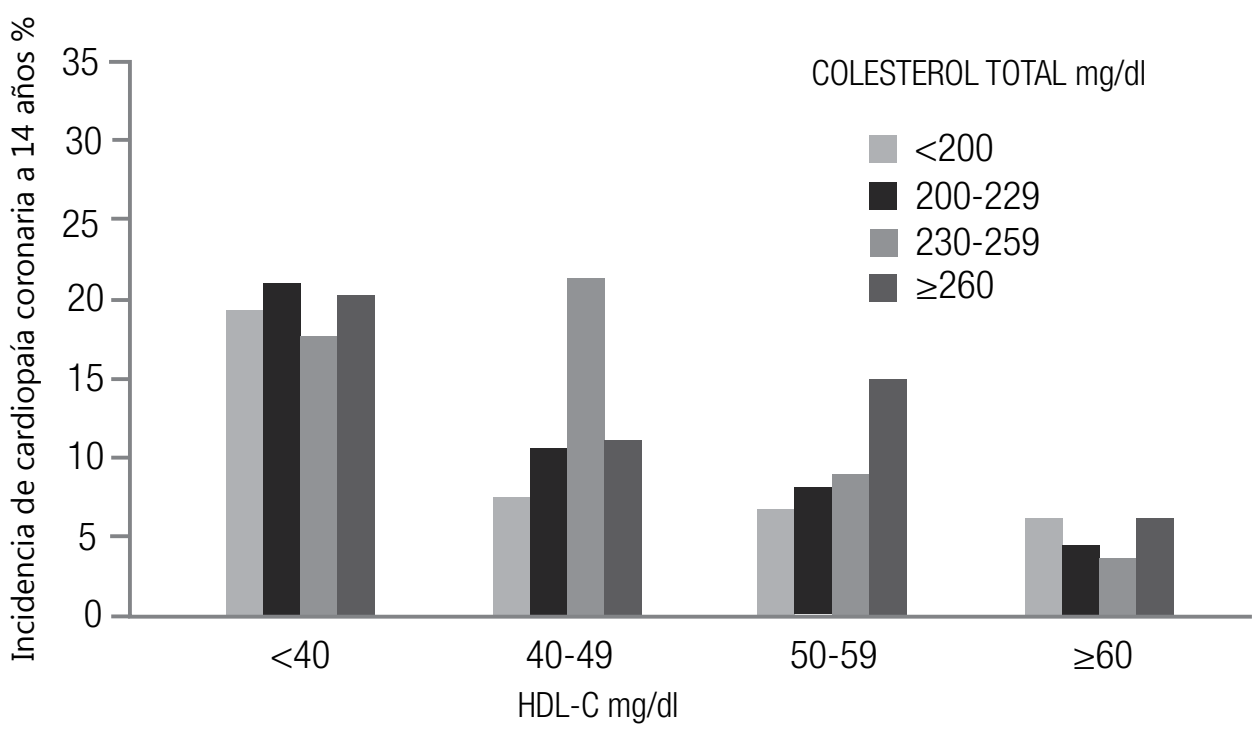

Adaptado de Castelli W. P et al. Incidence of coronary heart disease and lipoprotein cholesterol levels. The Framingham Study. JAMA 1986, 256: 2835-2838 
que la incidencia de cardiopatía coronaria no disminuía sustancialmente con el tiempo (Figura 1). En esta segunda determinación del HDL-C también se usó un modelo multivariado que incluyó tabaquismo, peso corporal, consumo de alcohol, glicemia, colesterol total y presión arterial. Se concluyó que aún después de estos ajustes persistía la relación inversa del HDL-C con el desarrollo de cardiopatía coronaria, tanto en hombres como en mujeres, con edades de 49 ańos y más, y que cualquier cambio de $10 \mathrm{mg}$ de HDL variaba el riesgo coronario en $50 \%{ }^{2,3,4}$.

Inicialmente se consideró, también basándose en el estudio PROCAM, que los niveles de $60 \mathrm{mg} / \mathrm{dl}$ o más de $\mathrm{HDL}$ eran protectores, y niveles inferiores a $40 \mathrm{mg} / \mathrm{dl}$, un factor peligroso 5 .

Sin embargo, investigaciones realizadas en los últimos años han comenzado a cuestionar esta relación lineal inversa entre los niveles de HDL y morbilidad o mortalidad cardiovascular. Existe consenso en relacionar los niveles bajos de HDL como un fuerte factor de riesgo independiente, que incluso se considera en las tablas para determinar el riesgo cardiovascular, pero el rol protector de los niveles muy altos de HDL está siendo cuestionado ${ }^{6,7}$.

De acuerdo a los estudios epidemiológicos, los niveles séricos asociados a mayor riesgo en hombres son menores a $40 \mathrm{mg} /$ $\mathrm{dl}$ y a menos de $48 \mathrm{mg} / \mathrm{dl}$ en las mujeres. Asimismo, se ha cuestionado el rol causal de HDL en la protección cardiovascular usando la randomización mendeliana ${ }^{8,9,10}$.

Un reciente estudio canadiense, CANHEART, ha evaluado a 631762 personas de 40 a 105 años de edad, que residían en Ontario el 1 de enero de 2008, excluyendo a: 1) los que tenían enfermedad cardiovas- cular (infarto de miocardio, insuficiencia cardiaca, accidente cerebrovascular, revascularización coronaria); 2) los que tenían comorbilidades (cáncer, demencia, enfermedad vascular periférica, aneurisma de aorta abdominal, trombosis venosa); y 3) los residentes en casas de reposo. Las metas de evaluación fueron la relación de niveles séricos de HDL colesterol y la mortalidad cardiovascular, la mortalidad por cáncer, y otras causas de mortalidad.

Los autores concluyen que los niveles de HDL colesterol se asocian a muchos factores, entre los que se encuentran los socioeconómicos, el estilo de vida y las comorbilidades. En cuanto a los estilos de vida, 5108 participantes en este estudio mostraron que los niveles más altos de HDL colesterol se asociaron a menor indice de masa corporal (IMC $<25 \mathrm{~kg} / \mathrm{m} 2$ ), actividad física moderada (> 30 minutos de caminata diaria), consumo de frutas y vegetales $(>=5$ porciones/día) y consumo de alcohol ( $>=5$ tragos por ocasión al menos una vez al mes durante el año anterior al estudio).

La población de los que tuvieron $<30$ $\mathrm{mg} / \mathrm{dl}$ de HDL pertenecían a un nivel socioeconómico más bajo; habían más hipertensos, diabéticos, fumadores, y más pacientes tenían enfermedad pulmonar obstructiva crónica (EPOC) que los que tuvieron $\mathrm{HDL}>90 \mathrm{mg} / \mathrm{dl}^{11}$.

Como puede apreciarse en la Figura 2, existe una curva en $U$ que relaciona niveles bajos y altos de HDL colesterol con la mortalidad, particularmente en hombres. Asimismo, se ha observado que una relación similar se presenta en la mortalidad cardiaca y no cardiaca. Esta investigación no fue diseñada para examinar otros aspectos del HDL colesterol, tales como tamaño de las partículas, subclases o función. 


\section{FIGURA 2}

\section{Mortalidad estandarizada por edad en hombres relacionada con niveles de HDL}

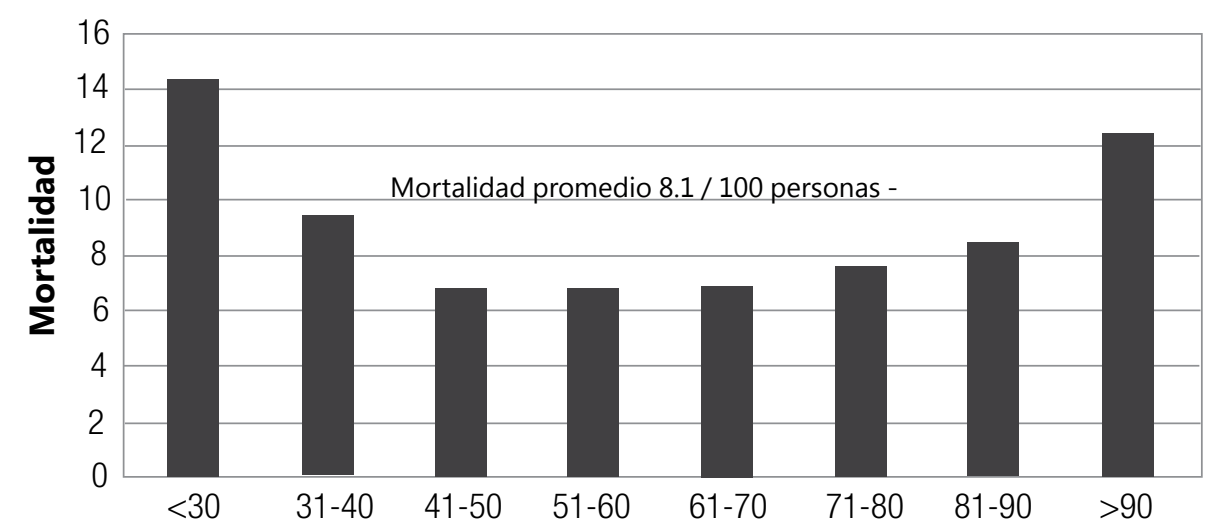

Adaptado de Canadian Cardiovascular Health in Ambulatory Care Research Team CANHEART AHA Clinical Science Special Report: Circ Cardiovasc Qual Outcomes. 2015;8(2):204-212. doi: 10.1161/CIRCOUTCOMES.114.001416.

\section{ESTUDIOS CON DROGAS}

La relación inversa entre los niveles de HDL y cardiopatía coronaria, detectada desde el punto de vista epidemiológico, llevó a la hipótesis de que al aumentar el HDL disminuiría la aterosclerosis coronaria. Esta hipótesis se reforzó con estudios experimentales en ratas en quienes al aumentar el HDL disminuía la placa aterosclerótica. Asimismo el tratamiento farmacológico con fibratos o con ácido nicotínico (niacina) mejoraba las cifras de HDL colesterol, pero en porcentajes no muy altos. De manera similar el dejar de fumar, hacer ejercicio, bajar de peso, contribuían a subir el HDL pero en porcentajes inferiores a $10 \%{ }^{12,13}$.

Sin embargo, los recientes estudios con niacina de liberación prolongada, o con fármacos que evitan el rubor facial, no mostraron disminución de la mortalidad.
El estudio AIM-HIGH (Tabla 1) enroló a pacientes que recibían simvastatina 40 a $80 \mathrm{mg} /$ día y si era necesario se les añadía ezetimibe $10 \mathrm{mg} /$ dia para mantener el nivel de LDL entre 40 a $80 \mathrm{mg} / \mathrm{dl}$. Un grupo (1718 pacientes) recibió además niacina 1500 a $2000 \mathrm{mg} /$ día y el grupo control (1696 pacientes) recibió placebo. El punto final fue el primer evento compuesto de muerte coronaria, infarto de miocardio no fatal, ACV isquémico, hospitalización por un síndrome coronario agudo o síntomas que requerirían revascularización coronaria o cerebral. El estudio se suspendió porque luego de tres años de seguimiento no hubo diferencias entre ambos grupos, A los dos ańos de tratamiento, el grupo de niacina aumentó el HDL de 35 a $42 \mathrm{mg} / \mathrm{dl}$, disminuyeron los triglicéridos de 164 a 122 $\mathrm{mg} / \mathrm{dl}$ y el LDL varió de 74 a $62 \mathrm{mg} / \mathrm{dl}$. El punto final ocurrió en 282 pacientes en el grupo de niacina y en 274 en el grupo control. La diferencia no fue estadísticamente 
TABLA 1

Estudio AIM HIGH

\begin{tabular}{|c|c|c|c|c|}
\hline Estudio AIM HIGH & Placebo & Niacina & IC $95 \%$ & $\mathbf{P}$ \\
\hline $\begin{array}{l}\text { Punto final primario } \\
\text { Mortalidad CV, IMA no } \\
\text { fatal, ACV isquémico, } \\
\text { hospitalización por SCA } \\
\text { o revascularización. }\end{array}$ & $\begin{array}{l}N=274 \\
(16.2 \%)\end{array}$ & $\begin{array}{l}N=228 \\
(16.4 \%)\end{array}$ & $1.02(0.87-1.21)$ & 0.80 \\
\hline $\begin{array}{l}\text { Mortalidad CV, IMA no } \\
\text { fatal, ACV isquémico, } \\
\text { SCA alto riesgo. }\end{array}$ & $\begin{array}{l}N=158 \\
(9.3 \%)\end{array}$ & $\begin{array}{l}N=171 \\
(10.0 \%)\end{array}$ & $1.08(0.87-1.34)$ & 0.49 \\
\hline $\begin{array}{l}\text { Mortalidad CV, IMA no } \\
\text { fatal, ACV isquémico. }\end{array}$ & $N=138$ & $N=156$ & $1.13(0.90-1.42)$ & 0.30 \\
\hline $\mathrm{ACV}$ isquémico. & $N=18$ & $N=20$ & $1.61(0.89-2.90)$ & 0.11 \\
\hline $\begin{array}{l}\text { Pacientes que requirie- } \\
\text { ron reducción de dosis. }\end{array}$ & $\begin{array}{l}N=58 \\
(3.4 \%)\end{array}$ & $\begin{array}{l}N=109 \\
(6.3 \%)\end{array}$ & & -0.001 \\
\hline $\begin{array}{l}\text { Pacientes que inte- } \\
\text { rrumpieron el fármaco. }\end{array}$ & $\begin{array}{l}N=341 \\
(20.1 \%)\end{array}$ & $\begin{array}{l}N=436 \\
(25.4 \%)\end{array}$ & & -0.001 \\
\hline
\end{tabular}

Adaptado de Atherothrombosis Intervention in Metabolic Syndrome with Low HDL/High Triglycerides: Impact on Global Health" (AIM-HIGH). N Engl J Med 2011;365:2255-2267 . doi: 10.1056/NEJMoa1 107579

significativa entre ambos grupos $(\mathrm{p}=079)$, lo que demostró que adicionar niacina al tratamiento establecido con la estatina, no contribuía a mejorar la morbi-mortalidad cardiaca o cerebral, en 36 meses, a pesar del aumento de $\mathrm{HDL}^{14}$.

Otro estudio, The Heart Protection Study 2-Treatment of HDL to Reduce the Incidence of Vascular Events (HPS-2 THRI$V E$ ), un ensayo de prevención secundaria que evaluó la adición de niacina de liberación prologada en pacientes que recibían tratamiento con estatinas, no mostró beneficio clínico comparado con los que recibieron solamente la estatina.

Después de 4 años de seguimiento de 25673 pacientes, el tratamiento con niacina más laropiprant (un fármaco que actúa contra el flush o rubor) no redujo el riesgo del punto combinado de muerte coronaria, infartos no fatales, accidentes cerebrovasculares, o revascularizaciones coronarias (14.5\%), comparado con los que recibían solamente estatinas $(15 \%)^{15}$.

\section{ESTRUCTURA Y FUNCIONES DE HDL COLESTEROL}

Con base en estudios sobre expresión y deleción de ABCA1, se ha determinado que el HDL-C se produce: $75 \%$ en el hígado, $20 \%$ en los intestinos y $5 \%$ en los macrófagos $^{16,17}$.

En los últimos años se ha profundizado el conocimiento del HDL y se ha mostrado que es una lipoproteína compleja que 
FIGURA 3

\section{Funciones del HDL Colesterol}

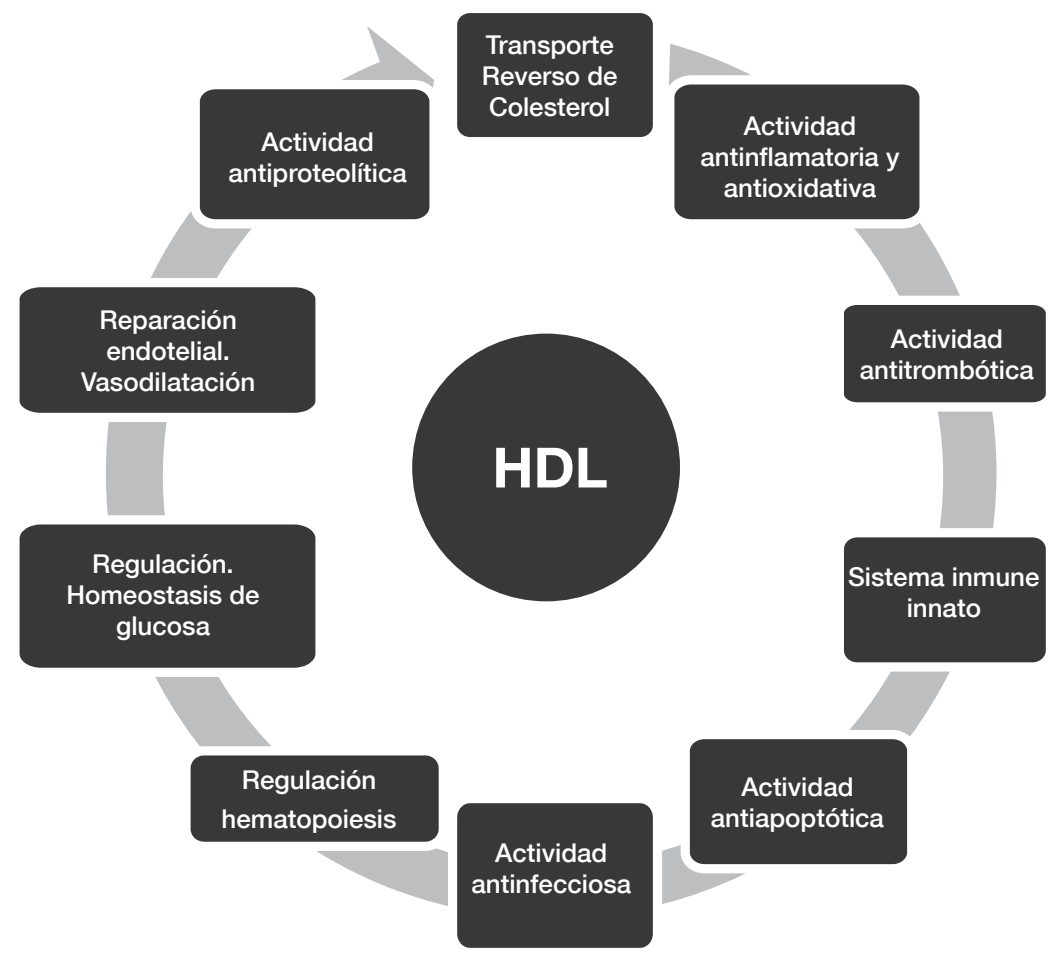

Adaptado de KA Rye, PJ Barter. Cardioprotective functions of HDLs. J Lipid Res, 2014; 55: $168-179$

consiste de varias subpoblaciones. Las HDL en el plasma humano son heterogéneas en términos de densidad, forma, carga eléctrica de la superficie, tamaño y composición. Desde el punto de vista de la densidad, se encuentran dos subfracciones: HDL2, que son partículas grandes y menos densas, y HDL3, que son pequeñas y más densas ${ }^{18,19}$.

Los estudios, asimismo, han identificado cinco subpoblaciones de HDL: $2 b$, $2 \mathrm{a}, 3 \mathrm{a} 3 \mathrm{~b}, \mathrm{y} 3 \mathrm{c}$, con una secuencia de disminución de tamaño que se acompaña de aumento de proteína y disminución progresiva de lípidos. Las HDL más grandes tienen apoAI, apoAII, apoE, apoCII y apoCIII, mientras que las pequeñas tienen apoAI, apoAII, apoAIV, apoF, apoJ, apoM,
apoL1,HRP, LpPLA2, PON1,PON3, PLTP, A1At, fibrinógeno, transtiretina ${ }^{20}$.

En virtud a sus diversos componentes, las HDL tienen una serie de funciones biológicas átero-protectoras y vásculo-protectoras, como puede apreciarse en la Figura 3. Estas funciones pueden variar en diversas condiciones como la inflamación, la diabetes mellitus, la uremia, y transformar a la HDL en una lipoproteína disfuncional, que se convierte en proaterosclerótica ${ }^{21}$.

\section{Transporte reverso de colesterol}

Con el propósito de elevar los niveles de HDL y reducir la morbi-mortalidad cardiovascular, se han ensayado fármacos con otros mecanismos de acción. La atención 
se ha centrado inicialmente en la inhibición de la cholesteryl ester transfer protein $(\mathrm{CETP})^{22}$.

El primer paso en el transporte reverso del colesterol (Figura 4) es la remoción de colesterol y fosfolípidos celulares de la pared vascular y de los tejidos extrahepáticos a la apoA-I, una lipoproteína importante en la HDL. La esterificación la realiza la LCAT, (lecithin colesterol acyl tranferase) que cambia a la HDL de la forma discoidal a la forma esférica. Estas partículas esterificadas son llevadas al hígado, donde son captadas por las SR-B1 ${ }^{23}$.

Asimismo, la CETP intercambia los triglicéridos de las lipoproteínas que contiene la apo lipoproteína apoB (LDL y VLDL) por esteres de colesterol en la HDL. Se ha reconocido a la CETP como un potente agente antiaterosclerótico, ya que en las personas con mutaciones de CETP se elevaban los niveles de $\mathrm{HDL}^{24}$.

El primer agente estudiado fue el torcetrapib. El estudio se realizó con 1188 pacientes coronarios que habían recibido atorvastatina para disminuir el LDL colesterol a menos de $100 \mathrm{mg} / \mathrm{dl}$ : un grupo fue randomizado a atorvastatina y el otro grupo a atorvastatina más el inhibidor de CETP, torcetrapib. Después de 24 meses, el grupo que recibió la combinación experimentó un importante aumento de $61 \%$ en HDL y una reducción de $20 \%$ en LDL, comparados con los que solo recibieron atorvastatina. Sin embargo, a pesar de estas grandes diferencias en los

\section{FIGURA 4}

Transporte reverso de colesterol

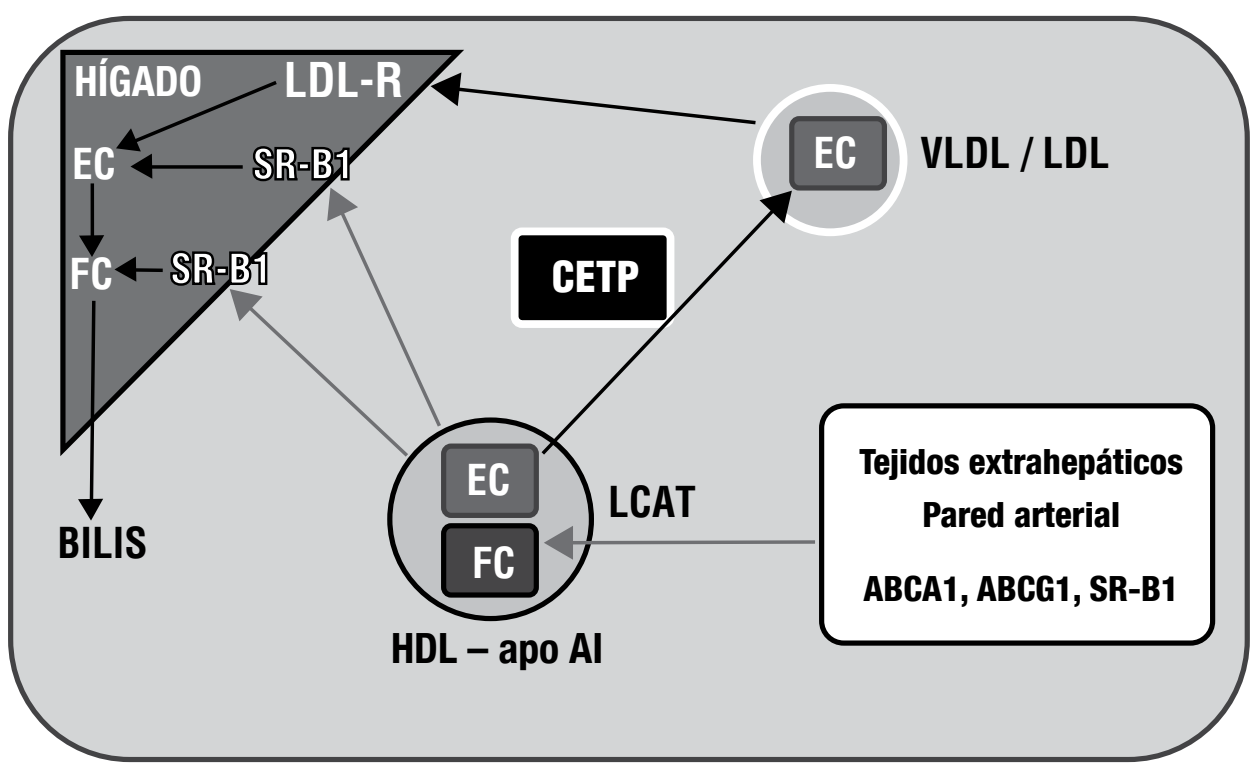

EC: Esteres de colesterol; SR-B1 receptor hepático de esteres de colesterol; LDL-R receptor de LDL; LCAT: lecitina colesterol acyl transferasa

Adaptado de Annema W et al. Regulation of reverse cholesterol transport - a comprehensive appraisal of available animal studies. Nutrition \& Metabolism 2012; 9:25 DOI: 10.1186/1743-7075-9-25(C) Annema and Tietge; licensee BioMed Central Ltd. 2012 
niveles de las lipoproteínas mencionadas, estos pacientes tuvieron un aumento de la presión arterial y no mostraron una disminución significativa en el volumen de la placa aterosclerótica ${ }^{25}$.

Los investigadores del estudio ILLumINATE evaluaron a 15067 pacientes con alto riesgo cardiovascular: a un grupo le administraron solo atorvastatina y al otro, atorvastatina más torcetrapib, para comparar el tiempo de aparición del primer evento cardiovascular que comprendía mortalidad coronaria, infarto de miocardio no fatal, ACV u hospitalización por angina inestable. Después de 12 meses, los pacientes del grupo que recibió torcetrapib registraron un aumento de $72,1 \%$ de HDL y una disminución de $24,9 \%$ de LDL, pero aumentó su presión sistólica en 5,4 mmHg; en ellos aumentó también el sodio, el bicarbonato y la aldosterona y disminuyó el potasio, en comparación con los que no recibieron este fármaco. Estos pacientes aumentaron su riesgo cardiovascular en forma muy considerable, y un análisis pos hoc exhibió un riesgo mayor de mortalidad. El grupo de investigación concluyó que la terapia con torcetrapib aumentó el riesgo de morbilidad y mortalidad por un mecanismo desconocido ${ }^{26}$.

Resultados similares se han encontrado con otro inhibidor de CETP, dalcetrapib. En un estudio que comprendió a 15871 pacientes con síndrome coronario agudo reciente, se randomizó a un grupo con dalcetrapib, y a otro, con placebo, además del tratamiento convencional. Los pacientes fueron seguidos por 31 meses: los que recibieron dalcetrapib

\section{TABLA 2}

Dallas Heart Study. HDL, factores de riesgo y partículas de HDL y eventos cardiovasculares

\begin{tabular}{l|c|c}
\multicolumn{1}{c}{ Modelos } & $\begin{array}{c}\text { \# participantes con eventos / } \\
\text { total de participantes }\end{array}$ & $\begin{array}{c}\text { Tasa de peligro / } \\
\text { IC95\% }\end{array}$ \\
\hline HDL colesterol & $132 / 2416$ & $0.64(0.40-1.01)$ \\
\hline Análisis no ajustado & & $\mathrm{N}=20$ \\
\hline Análisis ajustado & & $\mathrm{N}=109$ \\
\hline Para factores de riesgo tradicionales & & $(6.3 \%)$ \\
\hline $\begin{array}{l}\text { Para factores de riesgo tradicionales y } \\
\text { concentración de partículas HDL }\end{array}$ & & $132 / 2416$ \\
\hline Capacidad de eflujo de colesterol & & $0.30(0.27-0.73)$ \\
\hline Análisis ajustado & & $0.31(0.18-0.50)$ \\
\hline Para factores de riesgo tradicionales & & $0.34(0.31-0.52)$ \\
\hline Para factores de riesgo tradicionales y HDL & & $0.33(0.19-0.55)$ \\
\hline $\begin{array}{l}\text { Para factores de riesgo tradicionales y } \\
\text { concentración de partículas de HDL }\end{array}$ & & \\
\hline $\begin{array}{l}\text { Para factores tradicionales de riesgo, HDL } \\
\text { colesterol y concentración de partículas } \\
\text { de HDL }\end{array}$ & & \\
\hline
\end{tabular}

Adaptado de Rohatgi A et al. HDL cholesterol efflux capacity and incident cardiovascular events. Dallas Heart Study. N Engl J Med. 2014,;371(25):2383-93. doi: 10.1056/NEJMoa1409065. 
aumentaron sus niveles de HDL de $31 \%$ a $40 \%$, comparado con los que recibieron placebo, pero no redujeron el riesgo de eventos cardiovasculares recurrentes, por lo que el estudio fue suspendido ${ }^{27}$.

Los cambios bioquímicos que se han observado con torcetrapib, y algunos con dalcetrapib, no se han verificado con otros inhibidores como el anacetrapib, que tiene en camino un estudio de 30000 pacientes coronarios, cuyos resultados serán publicados en $2017^{28}$.

Sin embargo, el tercer inhibidor de CTP, evacetrapib, en el estudio ACCELERATE en 12000 pacientes ha tenido resultados similares a los dos primeros: un gran incremento de HDL por $130 \%$ y una reducción de $37 \%$ de LDL, pero no ha mostrado disminución en la morbimortalidad coronaria o cerebral ${ }^{29}$.

\section{CALIDAD VERSUS CANTIDAD DE HDL-C}

Una nueva interpretación sobre la importancia de HDL es que la calidad y no la cantidad de esta lipoproteína es la importante en la reducción del proceso aterosclerótico. Se ha postulado que el HDL puede transformarse en proaterosclerótica, sobre todo en situaciones con inflamación, y que ciertas subclases de HDL correlacionan mejor con los efectos antiateroscleróticos. Similar explicación se ha dado para interpretar que la inhibición de la CETP puede tener efectos anti- y proateroscleróticos y modificar la morbi-mortalidad, pero no se tiene claramente establecido los mecanismos detallados de acción. De un lado, inhibir la CETP disminuiría el LDL disponible para ser captado por los macrófagos que forman las células espumosas, un paso inicial en la formación de la placa (proceso antiaterosclerótico); y de otro lado, inhibiendo este proceso de remodelamiento del HDL pudieran disminuir la apo lipoproteína A-1 y los lugares de almacenamiento de HDL disponibles para el eflujo del colesterol periférico. Asimismo, se postula la posibilidad de que disminuyan los ésteres de colesterol que se transfieren a las lipoproteínas que contienen apo lipoproteína B que regresa al hígado para su excreción intestinal. Actualmente, la investigación de los nuevos fármacos que trabajan sobre el CETP intentan balancear estos dos efectos ${ }^{30}$.

En esta línea de pensamiento, se han diseñado estudios que pretenden dar respuesta a esta interrogante de si es la calidad, es decir la habilidad del HDL de aceptar el colesterol de los macrófagos, que es un paso muy importante en el transporte reverso de colesterol, y no la cantidad de HDL la que es cardioprotectora.

A 2924 pacientes libres de enfermedad cardiovascular, del Dallas Heart Study, se les midió la concentración de HDL, pero también la concentración de partículas de HDL y la capacidad de eflujo de colesterol. Estos pacientes fueron seguidos por 9,4 años para evaluar la aparición de enfermedad cardiovascular aterosclerótica - definida como el primer infarto agudo de miocardio-, ACV no fatal, revascularización coronaria o mortalidad por causas cardiovasculares.

Como se aprecia en la Tabla 2, los niveles séricos de HDL no correlacionaron con los eventos cardiovasculares, pero se encontró una reducción de $67 \%$ en el riesgo cardiovascular en el cuartil más alto de la capacidad de eflujo del colesterol, comparado con el cuartil más bajo. El estudio concluye que este marcador, que caracteriza el primer paso en el transporte reverso de colesterol, en un modelo ajustado -que incluyó los factores de riesgo cardiovascu- 
lar, los niveles séricos de HDL y la concentración de partículas-, está asociado de manera inversa con la incidencia de eventos cardiovasculares en esta cohorte poblacional y constituye un elemento adicional importante para entender el mecanismo vásculo-protector del $\mathrm{HDL}^{31}$.

Otro estudio prospectivo, EPIC-Norfolk, usando una técnica diferente, con colesterol radiomarcado, comparó la capacidad de eflujo de colesterol, en suero depletado de apoB, en 1745 pacientes coronarios y 1749 controles sanos. Los resultados mostraron una correlación inversa y significativa entre la capacidad de salida de colesterol y los eventos coronarios. Estos fueron independientes de los múltiples factores de riesgo coronario y se mantuvieron significativos después de ajustar los resultados para HDL colesterol y las concentraciones de apoA-I $\mathrm{I}^{32,33}$.
Adicionalmente a los ensayos farmacológicos, Voight et al. examinaron la relación entre los niveles de HDL y el riesgo cardiovascular, en dos análisis randomizados mendelianos. En el primero de ellos se evaluó el polimorfismo nucleótido simple (SNP) en el gen de la lipasa endotelial (LIPG Asn396Ser) en 20 estudios (20 913 casos de infarto de miocardio, 95407 controles); en el segundo análisis usaron como instrumento un score genético de 14 SNPs que se asocian exclusivamente con el HDL, y evaluaron este score en 12482 casos de infarto de miocardio y 41331 controles. Usaron como controles el score genético de 13 SNPs exclusivamente asociados con LDL.

Los portadores del alelo 396Ser tuvieron un HDL $13 \%$ más alto que los controles, pero no se asoció con menor riesgo de infarto de miocardio.

\section{FIGURA 5}

\section{Concentraciones plasmáticas de HDL colesterol en portadores versus no portadores del alelo Ser del polimorfismo Ser LIPG Asn396Ser}

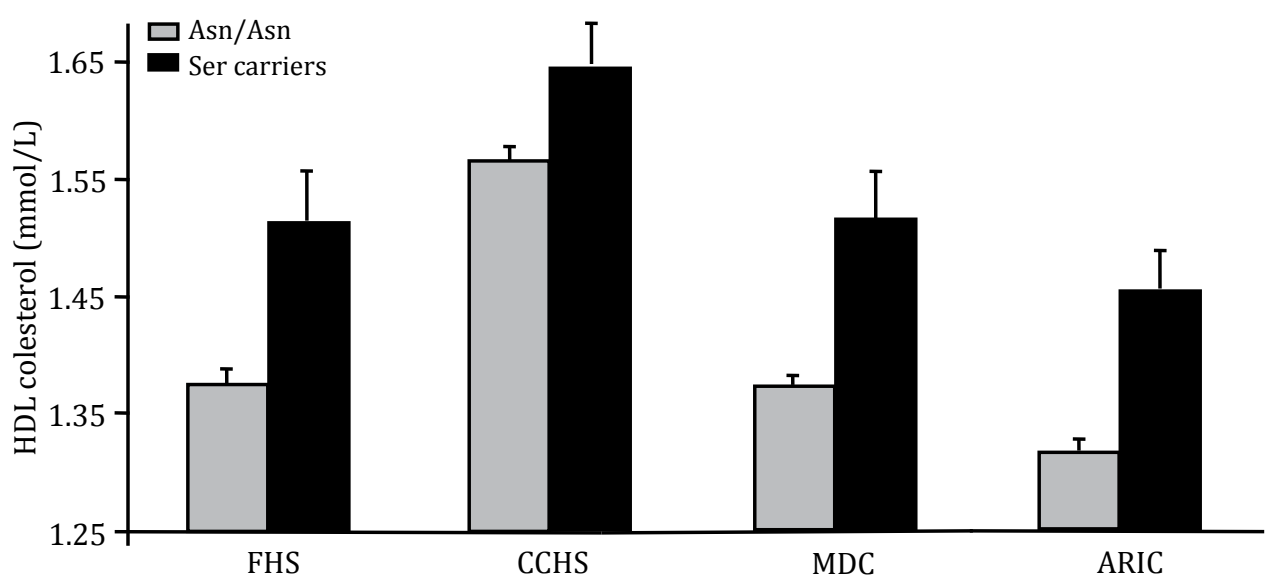

Voight BF et al. Plasma HDL cholesterol and risk of myocardial infarction: a mendelian randomisation study. Lancet. 2012;380(9841):572-80. doi: 10.1016/S0140-6736(12)60312-2.

FHS $=$ Framingham Heart Study. CCHS $=$ Copenhagen City Heart Study. MDC=Malmo Diet and Cancer Study. ARIC $=$ Atherosclerosis Risk in Communities Study. 
Igualmente, los polimorfismos en los 14 genes que tuvieron $\mathrm{HDL}$ más alto, sin cambios en los triglicéridos, tampoco mostraron una reducción en el riesgo cardiovascular. Es importante advertir que los autores interpretan estos resultados como que algunos mecanismos genéticos que elevan el HDL colesterol no parecen disminuir el riesgo de infarto de miocardio y que, asimismo, estos hallazgos cuestionan el concepto de que aumentando el HDL se producirán reducciones en el riesgo de infarto de miocardio ${ }^{34}$.

Un equipo de investigadores internacionales evaluó a 328 pacientes con niveles muy altos de HDL, con promedio de 107 $\mathrm{mg} / \mathrm{dl}$. En cinco de ellos encontraron una variante genética del gen SCARB1 que codifica para el mayor receptor de HDL en la célula hepática, el SR-BI (scavenger receptor class BI). La sobreexpresión de este gen disminuyó los niveles de HDL pero redujo la aterosclerosis. La depleción subió el HDL, pero aumentó la aterosclerosis.

Los análisis genéticos de más de 300000 personas confirmaron que la variante llamada SCARB1 P376L se asociaba a niveles altos de HDL, y para ver si se asociaba este hallazgo con cardiopatía coronaria se registraron datos de 50000 personas con cardiopatía coronaria y 88000 controles. Los investigadores encontraron que aquellos que tenían la variante genética tenían asimismo mayor riesgo de enfermedad cardiaca y concluyeron que los efectos protectores del HDL son más importantes por su funcionamiento que por su concentración ${ }^{35}$.

Algunos investigadores piensan que alguna de la evidencia que se ha presentado en contra de la hipótesis de la concentración de HDL pudiera ser explicada por haberse usado diferentes técnicas en la medición plasmática de HDL. Asimismo, apoAI parece jugar un papel importante en la prevención aterosclerótica y su nivel es inversamente proporcional a la enfermedad coronaria; su importancia es tan fuerte como el HDL. Similarmente, HDL-P, una medida de la concentración de la partícula de HDL, que es independiente de la concentración de HDL o de apoAI, puede predecir la cardiopatía coronaria igual o mejor que los niveles séricos de HDL o de apoAI ${ }^{36}$.

Mackey, en un estudio multiétnico, comparó las asociaciones independientes de las concentraciones HDL y de la partícula HDL-P con el grosor de la íntima-media de la carótida y la presencia de cardiopatía coronaria en 5598 hombres y mujeres de 45 a 84 años, sin enfermedad coronaria, $y$ encontró que tanto HDL-P como HDL estaban inversamente relacionados con el grosor de la relación íntima-media carotídea y de la enfermedad coronaria, pero la relación con los niveles séricos de HDL se debilitó cuando se ajustaron con los valores de LDL-P y HDL-P, demostrando que el HDL-P es un marcador más sensible para evaluar aterosclerosis carotídea y enfermedad coronaria incidental ${ }^{37}$.

\section{CONCLUSIONES}

Asistimos a una época desafiante en el conocimiento del rol que cumple el HDL en la aterogénesis y en el riesgo de la enfermedad cardiovascular.

Una serie de estudios epidemiológicos sugieren que el número de partículas puede ser superior al nivel sérico de HDL-C como meta terapéutica. Esta lipoproteína, que tiene una composición compleja, ejerce una diversa cantidad de acciones antiaterogénicas. La composición heterogénea de la lipoproteína HDL es también responsable de ejercer una amplia variedad de 
acciones protectoras contra la aterogénesis, y que son el reflejo de la amplia variedad de componentes lipídicos y proteicos que la forman ${ }^{38}$.

Cada vez hay más evidencia de que el número menor de partículas de HDL puede ser un mejor indicador del riesgo cardiovascular que los niveles séricos de HDL; en consecuencia, usar este parámetro puede orientar y ayudar a interpretar mejor la terapéutica ${ }^{39}$.

Hasta el momento, la capacidad antiaterosclerótica de las subpoblaciones del HDL no ha sido precisada clínicamente, pero la relación inversa entre el número de las HDL grandes y la cardiopatía coronaria sugiere que medir el perfil de la partícula de HDL puede ser útil ${ }^{40}$.

Debemos precisar que, en los momentos actuales, medir la concentración sérica de HDL sigue siendo un indicador importante, pero ańadir medidas independientes como apoAI, HDL-P y la función de esta lipoproteína, puede darnos mayores argumentos para predecir y disminuir el riesgo cardiovascular ${ }^{41}$.

\section{REFERENCIAS BIBLIOGRÁFICAS}

1. Stroes E. Statins and LDL cholesterol lowering: an overview. Curr Med Res Opin 2005;21 Suppl 6:: S9-S16 .doi.org/10.1185/0300799005X59102

2. Kannel WB, High-density lipoproteins: Epidemiologic profile and risks of coronary artery disease Amer J Cardiol 1983;52(4):B9-B12. doi. org/10.1016/0002-9149(83)90649-5.

3. Castelli WP et al. Incidence of coronary heart disease and lipoprotein cholesterol levels. The Framingham Heart Study. JAMA 1986;266:2835-2838.
4. Wong N. Nature Reviews Cardiology 2014;11:276-289 doi:10.1038/nrcardio.2014.26.

5. Assmann G et al. Simple Scoring Scheme for Calculating the Risk of Acute Coronary Events Based on the10-Year Follow-Up of the Prospective Cardiovascular Münster (PROCAM) Study. Circulation. 2002;105:310-315. doi: 10.1161/ hc0302.102575.

6. Stone NJ et al. F. 2013 ACC/AHA guideline on the treatment of blood cholesterol to reduce atherosclerotic cardiovascular risk in adults: a report of the American College of Cardiology/American Heart Association Task Force on Practice Guidelines. Circulation. 2014; 129: S1-S45. https://doi.org/10.1161/01. cir.0000437738.63853.7a.

7. Capano A et al. 2016 ESC/EAS Guidelines for the Management of Dyslipidaemias. The Task Force for the Management of Dyslipidaemias of the European Society of Cardiology (ESC) and European Atherosclerosis Society (EAS). Developed with the special contribution of the European Association for Cardiovascular Prevention \& Rehabilitation (EACPR) Atherosclerosis 2016; 253: 281-344. http:// dx.doi.org/10.1016/j.atherosclerosis.2016.08.018.

8. Sarwar $\mathrm{N}$ et al. Triglyceride-mediated pathways and coronary disease: collaborative analysis of 101 studies. Triglyceride Coronary Disease Genetics, Consortium and Emerging Risk Factors Collaboration. Lancet. 2010; 375: 1634-1639.

9. Holmes MV et al. Mendelian randomization of blood lipids for coronary heart disease. Eur Heart J. 2015; 36: 539-550

10. Harrison SC et al. Mendelian randomisation, lipids, and cardiovascular disease. Lancet. 2012;380:543-545 
11. Tu JV et al. The Cardiovascular Health in Ambulatory Care Research Team (CANHEART): using big data to measure and improve cardiovascular health and healthcare services. Circ Cardiovasc Qual Outcomes. 2015; 8(2):20412. doi: 10.1161/CIRCOUTCOMES.114.001416.

12. Russell JC. Of mice and men, rats and atherosclerosis Cardiovasc Res 2003; 59(4): 810 - 811. DOI: http://dx.doi. org/10.1016/S0008-6363(03)00530-3 810-811.

13. Calkin AC et al. Gemfibrozil decreases atherosclerosis in experimental diabetes in association with a reduction in oxidative stress and inflammation. Diabetologia (2006) 49: 766-774 doi 10.1007/ s00125-005-0102-6.

14. The AIM-HIGH Investigators. Niacin in Patients with Low HDL Cholesterol Levels Receiving Intensive Statin Therapy. N Engl J Med 2011;365:2255-2267 . doi: 10.1056/NEJMoa1 107579.

15. Armitage J et al. The Heart Protection Study 2-Treatment of HDL to Reduce the Incidence of Vascular Events (HPS2 THRIVE ), The HPS2-THRIVE Collaborative Group*. N Engl J Med 2014; 371:203-212 DOI: 10.1056/ NEJMoa1300955.

16. Highpassand M. et al. Monocyte/macrophage expression of $\mathrm{ABCA} 1$ has minimal contribution to plasma HDL levels. J Clin Invest. 2001, 108(9):1315-1320.

17. Timmins JM. Targeted inactivation of hepatic Abcal cuases profound hypoalphalipoproteinemia and kidney hypercatabolism of apoA1. J Clin Invest. 2005:115(5):1333-1342.

18. Rye KA, Clay MA, Barter PJ. Remodelling of high density lipoproteins by plasma factors. Atherosclerosis.1999; 145: 227-238.
19. Blanche PJ, Gong EL, Forte T. M, Nichols AV. Characterization of human high-density lipoproteins by gradient gel electrophoresis. Biochim. Biophys. Acta.1981;665: 408-419.

20. Davidson WS et al. Proteomic Analysis of Defined HDL Subpopulations Reveals Particle-Specific Protein Clusters. Relevance to Antioxidative Function. Arterioscler Thromb Vasc Biol 2009; 26(6):870-876.

21. Rye KA, Barter PJ. Cardioprotective functions of HDLs. J Lipid Res. 2014; 55: 168-179.

22. Khera AV, Cuchel M, De la Llera-Moya $\mathrm{M}$ et al. Cholesterol efflux capacity, high-density lipoprotein function, and atherosclerosis. N Engl J Med, 2011,364:. 127-135.

23. Annema $W$ et al. Regulation of reverse cholesterol transport-a comprehensive appraisal of available animal studies. Nutrition \& Metabolism 2012; 9:25 DOI: $10.1186 / 1743-7075-9-25 @$.

24. Lewis GF, Rader DJ: New insights into the regulation of HDL metabolism and reverse cholesterol transport. Circulation Research.2005; 96: 1221-1232. doi: 10.1161/01. RES.0000170946.56981.5c.

25. Nissen SE et al. Illustrate Investigators. Effect of Torcetrapib on the Progression of Coronary Atherosclerosis. N Engl J Med 2007; 356:1304-1316 DOI: 10.1056/NEJMoa070635.

26. Barter PJ et al. Illuminate Investigators. Effects of torcetrapib in patients at high risk for coronary events. N Engl J Med. 2007;357(21):2109-22.

27. Schwartz GG et al. For the dal-Outcomes Investigators* Effects of Dalcetrapib in Patients with a Recent Acute Coronary Syndrome. N Engl J Med 2012; 367:20892099 DOI: 10.1056/NEJMoa1206797. 
28. Cannon $\mathrm{C}$ et al. "Safety of Anacetrapib in Patients with or at High Risk for Coronary Heart Disease". The New England Journal of Medicine 2010363 (25): 2406-2415. doi:10.1056/NEJMoa1009744.

29. Nicholls $S$ et al. A Study of Evacetrapib in High-Risk Vascular Disease (ACCELERATE) 65th Annual Scientific Sesion 2016 del American College of Cardiology Chicago 2-4 abril 2016. https:// accscientificsession.acc.org/ accesado 15 mayo 2016.

30. Kerry-Anne Ryea, Kwok Leung Onga. HDL function as a predictor of coronary heart disease events: time to re-assess the HDL hypothesis? Lancet: Diabetes\&Endocrinology 2015;3(7):488489 http://dx.doi.org/10.1016/S22138587(15)00205-3.

31. Rohatgi A et al. HDL cholesterol efflux capacity and incident cardiovascular events. Dallas Heart Study. N Engl J Med. 2014,;371(25):2383-93. doi: 10.1056/NEJMoa1409065.

32. Arsenault BJ et al. HDL particle size and the risk of coronary heart disease in apparently healthy men and women: the EPIC-Norfolk prospective population study.Atherosclerosis2009;206:276281. doi:10.1016/j. atherosclerosis.2009.01.044.

33. El Harchaoui et al. High-density lipoprotein particle size and concentration and coronary risk. Ann. Intern. Med.2009; 150, 84-93. doi:10.7326/0003-4819150-2-200901200-00006.

34. Voight BF et al. Plasma HDL cholesterol and risk of myocardial infarction: a mendelian randomisation study. Lancet. 2012;380(9841):572-80. doi: 10.1016/S0140-6736(12)60312-2.
35. Zanoni $\mathrm{P}$, et al. Rare variant in scavenger receptor BI raises HDL cholesterol and increases risk of coronary heart disease. CHD Exome+ Consortium; CARDIoGRAM Exome Consortium; Global Lipids Genetics Consortium. Science. 2016;351(6278):1166-1171. doi: 10.1126/science.aad3517. PMID: 26965621.

36. Kontush A. HDL particle number and size as predictors of cardiovascular disease. Front. Pharmacol, 2015; 6:.218-224. doi 10.3389/fphar.2015.00218>. <hal01266148>.

37. Mackey E et al. High-Density Lipoprotein Cholesterol and Particle Concentrations, Carotid Atherosclerosis, and Coronary Events. MESA (Multi-Ethnic Study of Atherosclerosis) J Am Coll Cardiol. 2012;60(6):508-516. doi:10.1016/j.jacc.2012.03.060.

38. Matyus SP et al. HDL particle number measured on the Vantera(R), the first clinical NMR analyzer. Clin. Biochem. 2014;48: 148-155. doi:10.1016/j.clinbiochem.2014.11.017

39. Shah AS et al. Proteomic diversity of high density lipoproteins: our emerging understanding of its importance in lipid transport and beyond. J. Lipid Res.2013; 54, 2575-2585. doi: 10.1194/jlr.R035725.

40. Rosenson RS et al. HDL Measures, Particle Heterogeneity, Proposed. Nomenclature, and Relation to Atherosclerotic Cardiovascular Events. Clin. Chem. 2011; 57, 392-410. doi: 10.1373/clinchem.2010.155333),

41. Gary FL et al. New Insights Into the Regulation of HDL Metabolism and Reverse Cholesterol Transport. Circ Res. 2005;96:1221-1232 doi: 10.1161/. 